\title{
Human peripheral blood-derived exosomes for microRNA delivery
}

\author{
JI-YOUNG KANG ${ }^{1}$, HYEWON PARK $^{1}$, HYOEUN KIM $^{1}$, DASOM MUN $^{1}$, \\ HYELIM PARK $^{1}$, NURI YUN ${ }^{2}$ and BOYOUNG JOUNG ${ }^{1}$ \\ ${ }^{1}$ Division of Cardiology, Yonsei University College of Medicine; ${ }^{2}$ Institute of Life Science \\ and Biotechnology, Yonsei University, Seoul 03722, Republic of Korea
}

Received November 15, 2018; Accepted March 27, 2019

DOI: $10.3892 / \mathrm{ijmm} .2019 .4150$

\begin{abstract}
Exosomes serve important functions in cell-to-cell communication and biological functions by serving as a delivery cargo shuttle for various molecules. The application of an improved delivery method for microRNAs (miRNAs/miRs) may enhance their potential as a therapeutic tool in cardiac diseases. Thus, the present study investigated whether human peripheral blood-derived exosomes may be used as a delivery cargo system for miRNAs, and whether the delivery of miR-21 using a human peripheral blood derived-exosome may influence the degree of remodeling following myocardial infarction (MI). In H9C2 and HL-1 cells, miR-21 expression was successfully regulated by treatment with human peripheral blood derived-exosomes loaded with an miR-21 mimic or inhibitor compared with untreated cells. In addition, the mRNA and protein expression levels of SMAD family member 7 (Smad7), phosphatase and tensin homolog (PTEN) and matrix metalloproteinase 2 (MMP2), which are involved in cardiac fibrosis, were associated with the uptake of miR-21 mimic- or inhibitor-loaded exosomes. Similarly, the in vivo mRNA and protein expression of Smad7, PTEN and MMP2 were altered following treatment with miR-21 mimic- or inhibitor-loaded exosomes. Furthermore, miR-21 mimic-loaded exosomes enhanced fibrosis, whereas miR-21 inhibitor-loaded exosomes reduced fibrosis in a mouse MI model. These results suggested that miRNA-loaded human peripheral blood derived-exosomes may be used as a therapeutic tool for cardiac diseases.
\end{abstract}

Correspondence to: Professor Boyoung Joung, Division of Cardiology, Yonsei University College of Medicine, 250 Seungsanno, Seodaemun-gu, Seoul 03722, Republic of Korea

E-mail: cby6908@yuhs.ac

Professor Nuri Yun, Institute of Life Science and Biotechnology, Yonsei University, 50 Yonsei-ro, Seodaemun-gu, Seoul 03722, Republic of Korea

E-mail: yunnuri@hanmail.net

Abbreviations: LVEDV, left ventricular end-diastolic volume; LVIDD, left ventricular internal-diastolic diameter

Key words: exosome, human peripheral blood, microRNA, myocardial infarction, fibrosis

\section{Introduction}

Myocardial infarction (MI), also known as a heart attack, is one of the leading causes of mortality globally (1). Death of cardiomyocytes, angiogenesis, fibrosis, hypertrophy and contractile dysfunction during MI result in impaired cardiac function (2-5). In particular, cardiac fibrosis following MI serves a key function in regulating cardiac function (6). In addition, it has been identified as a key factor in the incidence of heart failure, as excessive fibrosis results in ventricular dilation, infarct expansion and hypertension (7). For this reason, inhibition of cardiac fibrosis progression in infarcted hearts may preserve cardiac function and prevent heart failure. However, at present, there is no efficient therapy available (8).

Exosomes, membrane-bound vesicles of 30-100 nm, are secreted by various cell types and are present in the majority of body fluids $(9,10)$. An exosome is a 'nanosphere' with a bilayered membrane, containing various types of lipids and proteins. For example, the majority of exosomes contain membrane transport and fusion proteins, heat-shock proteins, multivesicular body biogenesis proteins [including programmed cell death 6 interacting protein (Alix)], tetraspanin [including cluster of differentiation (CD)9, CD63 and CD81] and endosomal/lysosomal proteins [including lysosomal associated membrane protein 2 (LAMP2)]. In addition, exosomes are comprised of different types of lipids, including cholesterol, sphingolipids, phosphoglycerides, ceramides and saturated fatty acid chains. These exosome compositions function as cargo for cell-to-cell communication $(11,12)$. Lately, exosomes have emerged as a promising drug delivery system with lower toxicity compared with conventional delivery systems and high therapeutic efficacy (13). However, two of the major challenges in developing exosome-based drug delivery methods are the scale up procedure and utilization without side effects (14). As human peripheral blood is widely used for transfusion and is easily obtained, the use of human peripheral blood-derived exosomes as a microRNA (miRNA/miR) delivery system, has substantial potential as a therapeutic tool.

Previous studies have demonstrated that certain miRNAs function as essential modulators in a number of physiological and pathological MI processes, including fibrosis (15-17). Among various miRNAs, miR-21 serves a crucial role in the development of cardiac fibrosis in response to MI through numerous target genes, including SMAD family member 7 (Smad7), sprout RTK signaling antagonist 1 and phosphatase 
and tensin homolog (PTEN) (18-20). Thus, the function of miR-21 in regulating gene expression during fibrosis makes miR-21 an ideal candidate for therapeutic applications.

The present study aimed to investigate whether exosomes isolated from human peripheral blood may function as efficient vehicles for miRNA delivery. To achieve this aim, miR-21, a well-known therapeutic target for cardiac diseases, was transfected into human peripheral blood-derived exosomes and whether the exosomes may deliver the miRNA in in vitro and in vivo models was evaluated. Furthermore, whether exosomes loaded with an miR-21 inhibitor may modulate the fibrotic remodeling in an MI mouse model was investigated. Therefore, the present study may provide a potential, effective approach for the treatment of cardiac disease.

\section{Patients and methods}

Exosome isolation and characterization. Human peripheral blood was obtained from three patients without serious or progressive cardiac disease at Yonsei University Health System (Seoul, Korea) from August 2015 to July 2016; the clinical profile of the patients is presented in Table I. Written informed consent was obtained from all patients and the study protocol was ethically approved by the local ethics committee (Institutional Review Board of Severance Hospital (Seoul, Korea) of the Yonsei University Health System; approval no. YUMC 4-2011-0872). Exosomes were isolated from human peripheral blood using the Exoquick exosome precipitation kit (System Biosciences, Palo Alto, CA, USA) according to the manufacturer's protocol. Briefly, human peripheral blood was centrifuged at $3,000 \mathrm{x}$ g for $15 \mathrm{~min}$ at $4^{\circ} \mathrm{C}$ to remove cells and cellular debris. Exoquick Exosome Precipitation Solution (63 $\mu \mathrm{l}$ ) was added to $250 \mu \mathrm{l}$ supernatant, transferred to a sterile tube, and incubated for $12 \mathrm{~h}$ at $4^{\circ} \mathrm{C}$. Following incubation, the mixtures were centrifuged at $1,500 \mathrm{xg}$ for $30 \mathrm{~min}$ at $4^{\circ} \mathrm{C}$ and the supernatant was removed by aspiration. Exosome pellets were resuspended in $200 \mu$ l Dulbecco's phosphate-buffered saline (PBS), aliquoted to avoid freeze and thaw cycles, and stored at $-80^{\circ} \mathrm{C}$.

For transmission electron microscopy (TEM), exosomes were prepared in PBS. The samples were adsorbed to a Formvar-carbon-coated electron microscope grid (Leica Microsystems, Inc., Buffalo Grove, IL, USA) for $\sim 1 \mathrm{~min}$ and the grid surface was then touched for $15 \mathrm{sec}$ with $2 \%$ uranyl acetate. Exosome size and morphology were observed using a JEM-1011 electron microscope (JEOL Ltd., Tokyo, Japan).

Nanopaticle tracking analysis (NTA) was performed to measure the size distribution and concentration of exosomes using a NanoSight LM10 instrument (Malvern Instruments, Ltd., Malvern, UK). The samples were captured for $60 \mathrm{sec}$ at room temperature with a flow rate of $50 \mathrm{ml} / \mathrm{min}$, and the data was obtained using NTA v.3.2 software (Malvern Panalytical, Ltd., Malvern, UK), which allows videos of particles moving under Brownian motion to be captured and automatically analyzed to generate high-resolution size distribution and concentration data.

Isolated exosomes were labeled using the PKH26 Red Fluorescent Cell Linker kit (Sigma-Aldrich; Merck KGaA, Darmstadt, Germany) according to the manufacturer's protocol. Next, unlabeled or PKH26 red fluorescent dye-labeled exosomes were added to $\mathrm{H} 9 \mathrm{C} 2$ and HL-1 cell lines obtained from the American Type Culture Collection (ATCC, Manassas, VA, USA) and incubated for $24 \mathrm{~h}$ at $37^{\circ} \mathrm{C}$ in $5 \% \mathrm{CO}_{2}$. The cardiac cells were washed twice with PBS, fixed with $4 \%$ paraformaldehyde for $30 \mathrm{~min}$ at room temperature and stained with DAPI for $5 \mathrm{~min}$ at room temperature. The uptake of exosomes by cardiac cell lines was observed using a confocal microscope (Carl Zeiss, Jena, Germany).

miRNA transfection into exosomes. The negative control miRNA (cat. no. SMC-2003), miR-21 mimic (5'-UAGCUU AUCAGACUGAUGUUGA-3'; cat. no. SMM-002) and miR-21 inhibitor (5'-AUCGAAUAGUCUGACUACAACU-3'; cat. no. SMI-002) were obtained from Bioneer Corporation (Daejoen, Korea). The miRNAs were transfected into human peripheral blood-derived exosomes using the Exo-Fect ${ }^{\mathrm{TM}}$ Exosome Transfection kit (System Biosciences) according to the manufacturer's protocol. Next, the exosomes were washed twice using Amicon ultra centrifugation tubes (EMD Millipore, Billerica, MA, USA) with cold PBS. The transfected exosomes were directly used for further experiments.

Cell culture and hypoxia. $\mathrm{H} 9 \mathrm{C} 2$ cardiac muscle cells, purchased from the American Type Culture Collection (ATCC), were grown in Dulbecco's modified Eagle's medium (WelGENE, Inc., Daegu, Korea) supplemented with $10 \%$ fetal bovine serum (FBS; Young In Frontier Co., Ltd., Seoul, Korea) and $1 \%$ penicillin-streptomycin (Gibco; Thermo Fisher Scientific, Inc., Waltham, MA, USA). HL-1 cardiac muscle cells were obtained from ATCC. Cells were maintained in Complete Claycomb Medium (SAFC Biosciences, Lenexa, KS, USA) supplemented with $10 \%$ FBS, $1 \%$ penicillin-streptomycin, $100 \mu \mathrm{M}$ norepinephrine (Sigma-Aldrich; Merck KGaA) and $4 \mathrm{mM}$ L-glutamine (Gibco; Thermo Fisher Scientific, Inc.) in plates coated with $12.5 \mu \mathrm{g} / \mathrm{ml}$ fibronectin and $0.02 \%$ gelatin (both Sigma-Aldrich; Merck KGaA). To induce hypoxia, cells were placed in Forma ${ }^{\mathrm{TM}}$ Series II 3131 Water-Jacketed $\mathrm{CO}_{2}$ incubators (Thermo Fisher Scientific, Inc.) with $94 \% \mathrm{~N}_{2}, 5 \% \mathrm{CO}_{2}$ and $1 \% \mathrm{O}_{2}$ at $37^{\circ} \mathrm{C}$ for $24 \mathrm{~h}$. The corresponding normoxic control cells were maintained at $37^{\circ} \mathrm{C}$ in a humidified incubator with $5 \% \mathrm{CO}_{2}$ and $21 \% \mathrm{O}_{2}$. Following treatment with transfected exosomes into $\mathrm{H} 9 \mathrm{C} 2$ and $\mathrm{HL}-1$ cells for $24 \mathrm{~h}$ at $37^{\circ} \mathrm{C}$, the cells were exposed to hypoxia for a further $24 \mathrm{~h}$.

Reverse transcription-quantitative polymerase chain reaction $(R T-q P C R)$. Total RNA from cultured cells and mouse heart tissues was extracted using the miRNeasy Mini kit (Qiagen $\mathrm{GmbH}$, Hilden, Germany) according to the manufacturer's protocols. Complementary DNA was synthesized using the miRNA 1st-Strand cDNA Synthesis kit (Agilent Technologies, Inc., Santa Clara, CA, USA) and the High Capacity cDNA Reverse Transcription kit (Applied Biosystems; Thermo Fisher Scientific, Inc.) according to the manufacturer's protocol. RT-qPCR was performed on an AriaMx Realtime PCR System using the Brilliant III Ultra-Fast SYBR ${ }^{\circledR}$-Green QPCR Master Mix (both Agilent Technologies, Inc.). The reaction conditions were as follows: $95^{\circ} \mathrm{C}$ for $3 \mathrm{~min}$ followed by 40 cycles at $95^{\circ} \mathrm{C}$ for $3 \mathrm{sec}$ and $55^{\circ} \mathrm{C}$ for $20 \mathrm{sec}$. The PCR primers were synthesized by Cosmo Genetech Co., Ltd. (Daejeon, Korea) and are listed in Table II. The relative expression levels of the miRNAs and the 
Table I. Clinical profile of patients from whom exosomes were isolated.

\begin{tabular}{llll}
\hline Case & Sex/age & \multicolumn{1}{c}{ Diagnosis } & Medication \\
\hline 1 & Female/66 & Atrial flutter & Amiodarone \\
2 & Female/77 & Atrial flutter, hypertension & Verapamil \\
3 & Male/67 & Paroxysmal supraventricular tachycardia, hypertension & Perindopril
\end{tabular}

Table II. Primers used for reverse transcription-quantitative polymerase chain reaction.

\begin{tabular}{lll}
\hline Gene name & \multicolumn{1}{c}{ Forward sequence (5'-3') } & Reverse sequence (5'-3') \\
\hline $\begin{array}{l}\text { mmu-miR-21 } \\
\text { mmu-U6 }\end{array}$ & GGGGTAGCTTATCAGACTGATG & \\
mmu-Smad7 & GCTTCGGCAGCACATATACTAAAAT & GATGGAGAAACCAGGGAACA \\
mmu-PTEN & GTGGCATACTGGGAGGAGAA & AGGGGTAGGATGTGAACCAGTA \\
mmu-MMP2 & GAGGGATAAAACACCATG & ACAGGAAGGGGAACTTTGAGTA \\
mmu-Collagen I & CACATACAGGATCATTGGTTACAC & GCAGCTGACTTCAGGGATGT \\
mmu- Collagen III & CATGTTCAGCTTTGTGGACCT & TGAGTCGAATTGGGGAGAAT \\
mmu-GAPDH & TCCCCTGGAATCTGTGAATC & CCATTTTGTCTACGGGACGA \\
rno-miR-21 & GGGTTCCTATAAATACGGACTGC & \\
rno-U6 & CGCCGTAGCTTATCAGACTG & \\
rno-Smad7 & CTCGCTTCGGCAGCACA & GAGGCAGTAAGACAGGGATGA \\
rno-PTEN & TTTTGAGGTGTGGTGGG & GTCCTTACTTCCCCAT \\
rno-MMP2 & AGAACTTATCAAACCCTT & AAAGGCAGCGTCTACTTGCT \\
\hline
\end{tabular}

miR, microRNA; Smad7, SMAD family member 7; PTEN, phosphatase and tensin homolog; MMP2, matrix metalloproteinase 2.

miR-21 target genes were calculated using the $2^{-\Delta \Delta \mathrm{Cq}}$ method (21) and normalized against U6 and GAPDH, respectively.

Western blot analysis. Total protein from cultured cells and mouse heart tissues was extracted with radioimmunoprecipitation assay Lysis and Extraction Buffer containing Protease and Phosphatase Inhibitor Cocktail (both Thermo Fisher Scientific, Inc.). The protein concentration of cell and tissue lysates was determined using a Pierce ${ }^{\mathrm{TM}}$ 660 nm Protein Assay reagent (Thermo Fisher Scientific, Inc.). Equal amounts of cell $(20 \mu \mathrm{g})$ and tissue lysates $(60 \mu \mathrm{g})$ were separated on a $10 \%$ Gradi-Gel $^{\mathrm{TM}}$ II gradient gel (Elpis Biotech, Inc., Daejeon, Korea) and transferred to polyvinylidene difluoride membranes (EMD Millipore, Billerica, MA, USA). The membranes were subsequently blocked for $1 \mathrm{~h}$ at room temperature in tris-buffered saline (TBS)-0.1\% Tween 20 (TBS-T) containing 5\% bovine serum albumin and incubated overnight at $4^{\circ} \mathrm{C}$ with primary antibodies against the following proteins: Alix (cat. no. sc-53540; 1:1,000), LAMP2 (cat. no. sc-18822; 1:1,000; both Santa Cruz Biotechnology Inc., Dallas, TX, USA), CD9 (cat. no. EXOAB-CD9A-1; 1:1,000), CD63 (cat. no. EXOAB-CD63A-1; 1:1,000; both System Biosciences), CD81 (cat. no. sc-166029; 1:1,000), Smad7 (cat. no. sc-365846; 1:1,000), PTEN (cat. no. sc-7974; 1:1,000; all Santa Cruz Biotechnology Inc.), matrix metalloproteinase 2 (MMP2; cat. no. ab37150; 1:1,000; Abcam, Cambridge, UK) and GAPDH (cat. no. sc-166574; 1:1,000; Santa Cruz
Biotechnology Inc.). GAPDH was used as the loading control. Following three washes with TBS-T, the membranes were incubated with horseradish peroxidase-conjugated goat anti-mouse IgG-HRP (cat. no. sc-2005; 1:5,000) and goat anti-rabbit IgG-HRP (cat. no. sc-2004; 1:5,000; both Santa Cruz Biotechnology Inc.) for $1 \mathrm{~h}$ at room temperature. Blotted proteins were visualized using an enhanced chemiluminescence kit (Santa Cruz Biotechnology Inc.).

Animal experiments. A total of 20 adult male C57BL/6 mice (8 weeks old; 20-25 g) were purchased from the Orient Bio Inc. (Seongnam, Korea). All mice were maintained under standard conditions (temperature, $20 \pm 0.5^{\circ} \mathrm{C}$; humidity, $60 \pm 5 \%$; light/dark cycle, $12 \mathrm{~h}$ ), and allowed free access to food and water. The mice were anesthetized by intraperitoneal injections of ketamine $\left(100 \mathrm{mg} / \mathrm{kg}\right.$ ) and xylazine (Rompun ${ }^{\circledR}$, $10 \mathrm{mg} / \mathrm{kg}$ ) and maintained with a positive pressure ventilation using a ventilator (Harvard Apparatus Co., Millis, MA, USA). MI was then induced by ligation of the left anterior descending coronary artery with a 6-0 silk suture (Ethicon, Inc., Cincinnati, OH, USA). Prior to injecting into the MI mice, negative control miRNA, miR-21 mimic or miR-21 inhibitor at a final concentration of $5 \mu \mathrm{mol} / \mathrm{ml}$ were loaded into a $200 \mu \mathrm{l}$ exosome sample containing $1 \mu \mathrm{g} / \mu \mathrm{l}$ exosomal proteins using the Exoquick exosome precipitation kit (System Biosciences) according to the manufacturer's protocol. Next, exosomes containing negative control miRNA, miR-21 mimic 
or inhibitor were administered by three intra-myocardial injections (total $100 \mu \mathrm{l}$ ) into the infarct border zone, as previously described $(22,23)$. The mice were divided into the following five groups (four mice/group): i) non-MI, ii) MI, iii) $\mathrm{MI}+$ negative control miRNA-loaded exosome injection, iv) $\mathrm{MI}+$ miR-21 mimic-loaded exosome injection and v) MI + miR-21 inhibitor-loaded exosome injection. Cardiac tissues were obtained from the left ventricular (LV) infarcted zone, infarct border zone and remote zone in the MI mice and from the LV apex in the non-MI mice. All procedures were performed in accordance with the ethical approval of the Institutional Animal Care and Use Committee of Yonsei University College of Medicine (approval reference no. 2011-0136) and the Guide for the Care and Use of Laboratory Animals published by the National Institutes of Health (24).

Echocardiography was performed with a Vevo 2100 system (VisualSonics, Inc., Toronto, ON, Canada). LV ejection fraction (LVEF) and fractional shortening (FS) were calculated according to the following formulas: LVEF $(\%)=[$ left ventricular end-diastolic volume (LVEDV)-left ventricular end-systolic volume /LVEDV) x100; left ventricular fractional shortening (LVFS) $(\%)=$ left ventricular internal-diastolic diameter (LVIDD)-left ventricular internal-systolic diameter)/LVIDD x100.

The mice were sacrificed one week following the treatment and the cardiac tissue were quickly collected and fixed in $4 \%$ formaldehyde for seven days at room temperature. Tissues were embedded in paraffin and sliced into $4 \mu \mathrm{m}$ thick sections. Next, the sections were mounted on normal glass slides and stained with hematoxylin and eosin (H\&E) for 2 min at room temperature and Masson's Trichrome (MT) for $10 \mathrm{~min}$ at room temperature. Four hearts were analyzed in each group and five distinct fields were examined in each slide, resulting in the evaluation of 20 fields in total from each group. Each slide was examined using an optical microscope and quantified with ImageJ 1.50i software (National Institutes of Health, Bethesda, MD, USA).

Statistical analysis. The data are presented as the mean \pm standard deviation $(n=4)$. The comparisons between the experimental groups were conducted using either an unpaired two-tailed Student's t-test, or a one-way analysis of variance with a Student-Newman-Keuls post hoc test. Statistical analyses were performed using SPSS version 23.0 (SPSS, Inc., Chicago, IL, USA) and GraphPad Prism 5 (GraphPad Software, Inc., La Jolla, CA, USA). P<0.05 was considered to indicate a statistically significant difference.

\section{Results}

Characterization of human peripheral blood-derived exosomes. Exosomes isolated from human peripheral blood were examined by TEM, NTA and western blot analysis. TEM analysis revealed that the isolated exosomes had a typical round morphology (Fig. 1A) and NTA revealed that the isolated exosomes had a mean particle size distribution of $104 \mathrm{~nm}$ (Fig. 1B). Consistent with these results, western blot analysis demonstrated the presence of known exosome markers, including Alix, LAMP2, CD9, CD63 and CD81, in the exosomes. The exosomes revealed high expression of markers, but GAPDH was absent (Fig. 1C). The detection of shape, size and protein markers of typical exosomes indicated that the exosomes were successfully isolated from human peripheral blood. To further investigate whether human peripheral blood-derived exosomes were taken up by cardiac cell lines, the exosomes with were labelled with PKH26, a red florescent dye. After $24 \mathrm{~h}$ treatment of H9C2 and HL-1 cells with labeled exosomes, a positive PKH26 signal was validated using confocal microscopy (Fig. 1D). These observations suggest that human peripheral blood-derived exosomes were effectively absorbed in the two cardiac cell lines.

Exosome-mediated delivery of miRNA in vitro. In order to determine the miRNA delivery potential of human peripheral blood derived-exosomes in vitro, exosomes were loaded with either miR-21 mimic or inhibitor using the Exo-Fect ${ }^{\mathrm{TM}}$ Exosome Transfection reagent (Fig. 2A). Following treatments with miR-21 mimic- or inhibitor-loaded exosomes in $\mathrm{H} 9 \mathrm{C} 2$ and HL-1 cells, RT-qPCR was performed to measure the levels of miR-21. Under normoxic conditions, miR-21 expression levels in H9C2 and HL-1 cells were significantly upregulated following treatment with miR-21 mimic-loaded exosomes compared with normoxic controls $(\mathrm{P}<0.01$; Fig. $2 \mathrm{~B}$ and $\mathrm{F})$. Next, it was detected that miR-21 expression was upregulated in hypoxic cardiac cells compared with normoxic controls and that miR-21 expression was regulated by the presence of its mimic and inhibitor when compared to the hypoxic controls. Under the conditions of hypoxic H9C2 cells, miR-21 mimic and inhibitor significantly upregulated and downregulated miR-21 expression compared with hypoxic controls, respectively $(\mathrm{P}<0.05$; Fig. $2 \mathrm{C})$. Similarly, in hypoxic HL-1 cells, miR-21 mimic-loaded exosomes significantly increased miR-21 expression compared with the normoxic and hypoxic controls $(\mathrm{P}<0.01)$, whereas miR-21 inhibitor-loaded exosomes significantly reduced miR-21 expression ( $\mathrm{P}<0.005$; Fig. 2G). Collectively, these observations suggest that human peripheral blood-derived exosomes effectively delivered miR-21 mimic or inhibitor to the two cardiac cell lines under all tested conditions.

It is well known that miRNAs exert their functions via the suppression of their target gene expression by binding to the 3'-untranslated region of target mRNAs $(25,26)$; thus, the present study next examined the mRNA and protein expression levels of Smad7, a direct target of miR-21, previously implicated in fibrosis regulation $(19,27)$. It was observed that treatment with miR-21 mimic-loaded exosomes in H9C2 cells significantly downregulated the mRNA and protein expression levels of Smad7 compared with hypoxic controls and that the expression of Smad7 was significantly upregulated in cells treated with miR-21 inhibitor-loaded exosomes $(\mathrm{P}<0.05$; Fig. 2D and E). Similarly, in HL-1 cells, hypoxia induced the significantly reduced expression levels of Smad7 mRNA and protein compared with normoxic controls, which were downregulated by miR-21 mimic- and upregulated by miR-21 inhibitor-loaded exosomes $(\mathrm{P}<0.05$; Fig. $2 \mathrm{H}$ and $\mathrm{I})$. Altogether, these results demonstrate that miR-21 mimic- or inhibitor-loaded exosomes were effectively delivered into cardiac cells and exhibited therapeutic potential by regulating target genes associated with physiopathological mechanisms. 
A

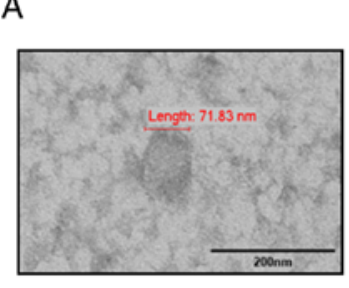

B

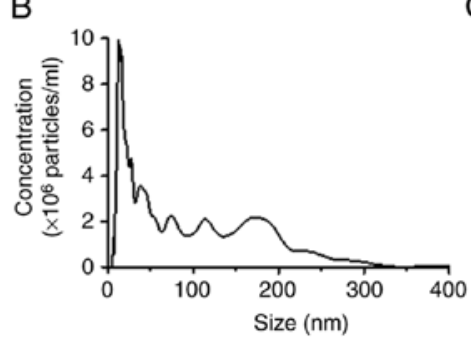

C $\mathrm{H}_{9 \mathrm{C} 2} \mathrm{HL}-1 \quad$ Exo

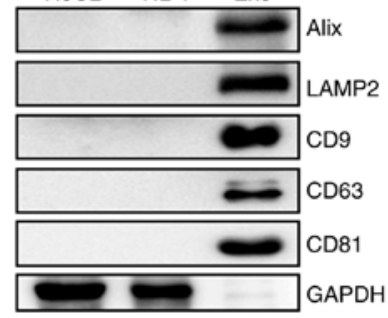

D
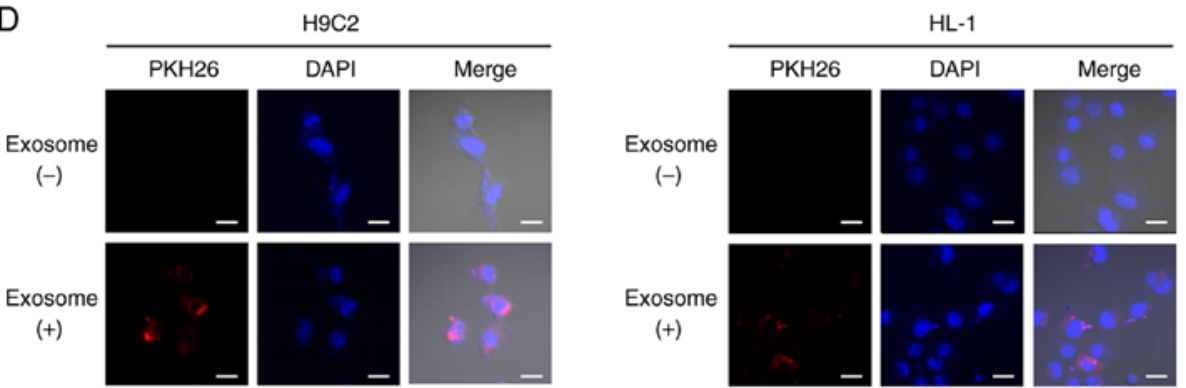

Figure 1. Characterization of exosomes isolated from human peripheral blood and uptake of exosomes by cardiac cells. (A) Morphology of human peripheral blood-derived exosomes imaged using transmission electron microscopy (scale bar, $200 \mathrm{~nm}$ ). (B) Human peripheral blood particle size/concentration were analyzed by nanoparticle tracking analysis. (C) Protein expression were determined using western blot analysis using the indicated exosome marker antibodies. (D) H9C2 and HL-1 cells were treated with unlabeled or PKH26 (red)-labeled exosomes for $24 \mathrm{~h}$ and analyzed by confocal microscopy (scale bar, $20 \mu$ m; magnification, x800). Cell nuclei were stained with DAPI (blue). Alix, programmed cell death 6 interacting protein; LAMP2, lysosomal associated membrane protein 2; CD, cluster of differentiation.

Regulation offunctional target using miRNA-loaded exosomes. Furthermore, the present study assessed the expression of PTEN, a well-known direct target of miR-21 (28), in order to validate the efficiency of exosomes as a delivery system. PTEN is expressed in various cell types, including cardiomyocytes, fibroblasts, endothelial cells and vascular smooth muscle cells, where it modulates cell survival/apoptosis, hypertrophy, contractility, fibrosis and metabolism $(28,29)$. Hence, PTEN is a crucial molecule involved in the development of numerous cardiovascular diseases (30). It was observed that miR-21 mimic-loaded exosomes significantly suppressed PTEN expression compared with hypoxic controls, whereas miR-21 inhibitor-loaded exosomes significantly promoted PTEN expression compared with H9C2 and HL-1 cells treated with miR-21 mimic-loaded exosomes $(\mathrm{P}<0.05$; Fig. 3A-D). In addition, previous reports have demonstrated that miR-21-regulated expression of MMP-2 is modulated via a PTEN pathway and results in cardiac fibrosis $(18,31)$. In the two cell lines, miR-21 mimic-loaded exosomes significantly increased MMP-2 expression compared with hypoxic controls, whereas miR-21 inhibitor-loaded exosomes significantly reduced MMP-2 expression compared to cells treated with miR-21 mimic-loaded exosomes $(\mathrm{P}<0.05$; Fig. 3A-D).

Exosome-mediated delivery of miRNA in vivo. C57BL/6 mice were subjected to MI to evaluate miR-21 expression levels in the infarcted, border and remote zones. MiR-21 expression levels were significantly upregulated in the MI mouse model, compared with non-MI mice, particularly in the infarcted zone of the heart $(\mathrm{P}<0.05$; Fig. 4A). Next, the expression levels of Smad7, PTEN and MMP2 were assessed using RT-qPCR and western blot analysis to determine whether miR-21 mimic- or inhibitor-loaded exosomes were delivered efficiently in vivo.
Similar to the results of in vitro experiment, it was observed that the mRNA and protein expression levels of Smad7, PTEN and MMP2 were significantly altered following treatment with miR-21 mimic-loaded exosomes when compared with mice treated with inhibitor-loaded exosomes $(\mathrm{P}<0.05$; Fig. $4 \mathrm{~B}$ and $\mathrm{C})$.

Therapeutic potential of miRNA-loaded exosomes against cardiac fibrosis. Finally, the present study investigated the potential of human peripheral blood-derived exosomes to deliver miRNA for the treatment of cardiac fibrosis following MI. The echocardiography analysis revealed worse cardiac function in mice treated with miR-21 mimic-loaded exosomes compared with mice treated with miR-21 inhibitor-loaded exosomes, as the former group of mice exhibited significantly decreased LVEF and LVFS compared with the latter group of mice $(\mathrm{P}<0.05$; Fig. $5 \mathrm{~A})$. As observed following the $\mathrm{H} \& \mathrm{E}$ and MT staining, myocardial disorders and fibrosis were significantly enhanced in MI mice compared with non-MI mice $(\mathrm{P}<0.05)$. Histological examination of the hearts of mice treated with miR-21 mimic-loaded exosomes revealed the dilatation of the left ventricular chambers with thinning of the walls. However, the histological changes were reduced following treatment with miR-21 inhibitor-loaded exosomes. In addition, cardiac fibrosis at the infarcted and border zones increased in mice treated with miR-21 mimic-loaded exosomes compared with MI mice, and this effect was reversed by treatment with miR-21 inhibitor-loaded exosomes (Fig. 5B and C).

Furthermore, the expression levels of myocardial fibrosis markers, collagen I and III, were significantly upregulated in MI mice compared with non-MI mice. Mice treated with miR-21 mimic- or inhibitor-loaded exosomes significantly upregulated and downregulated collagen I and III expression levels, respectively $(\mathrm{P}<0.05$; Fig. 5D). Collectively, 

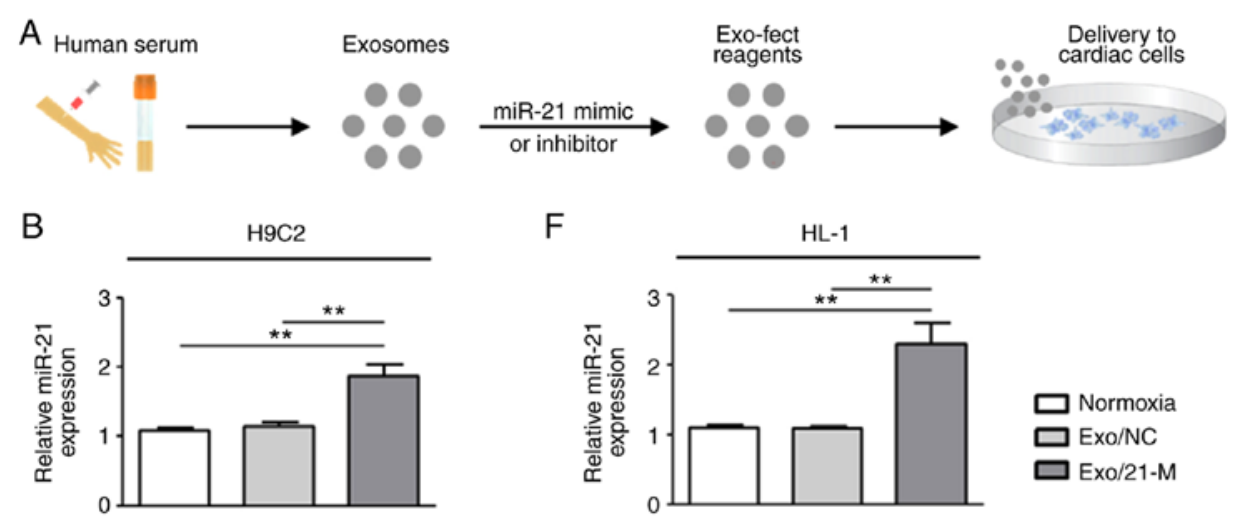

$\mathrm{F}$
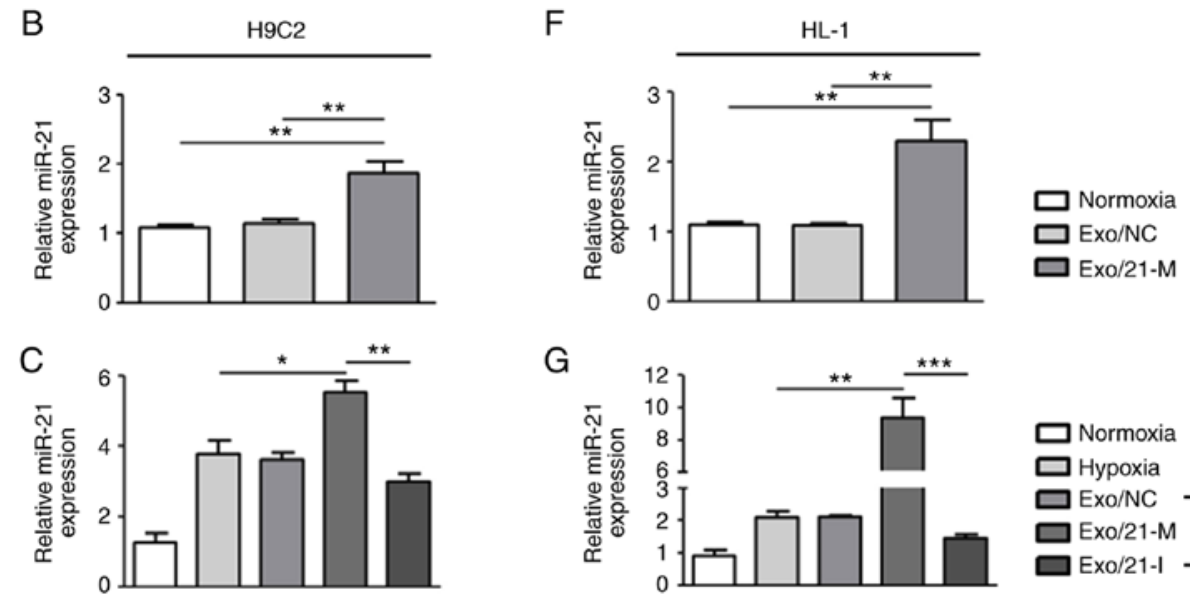

G
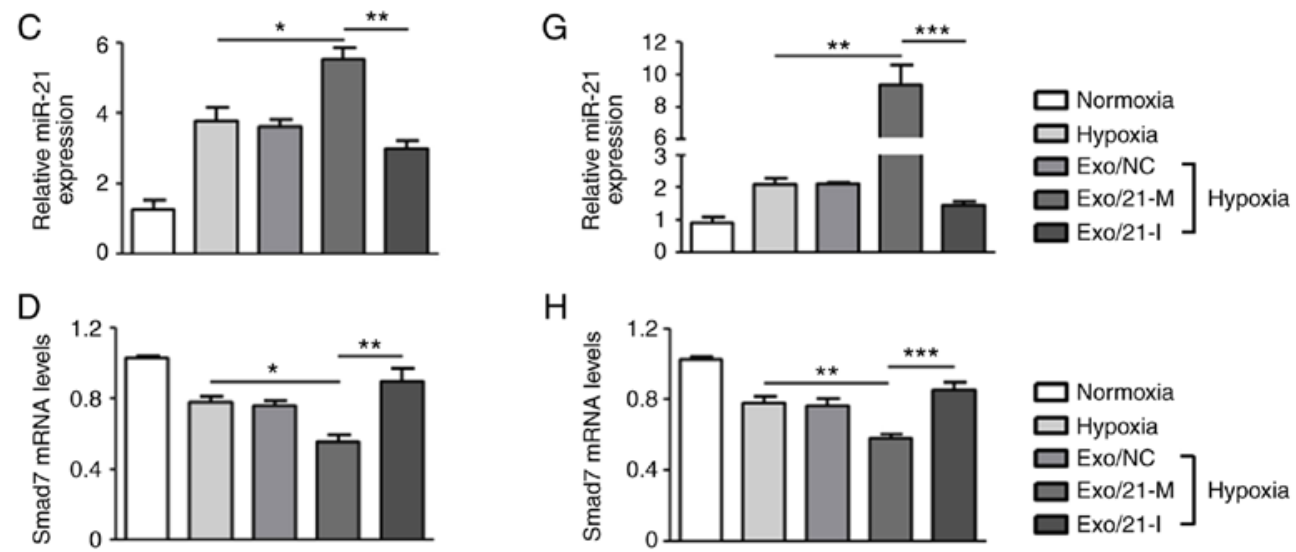

$\mathrm{H}$

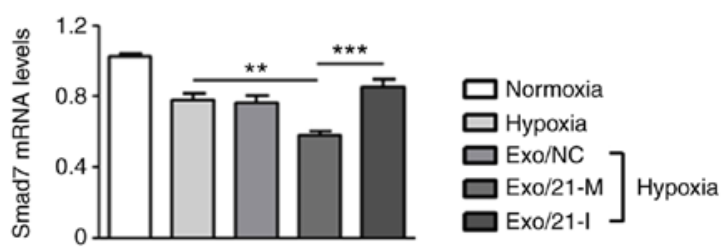

$\mathrm{E}$

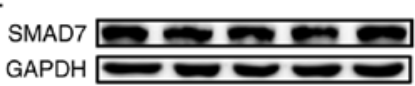

।
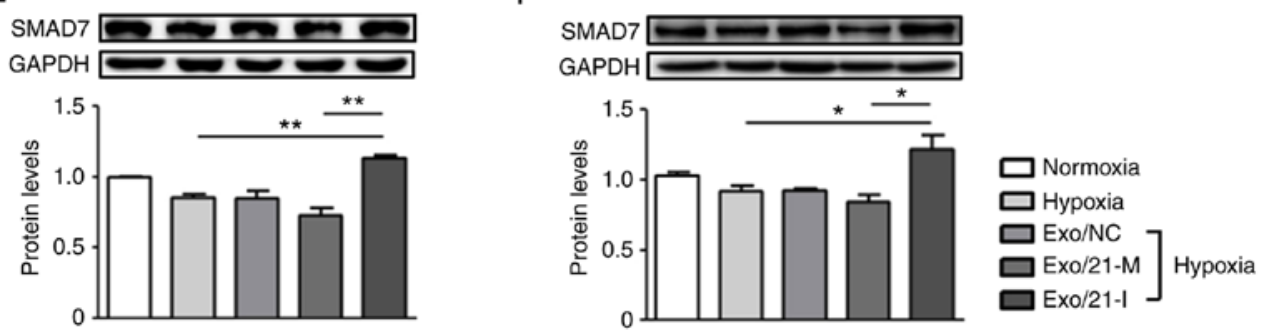

Figure 2. Delivery of miR-21 mimic- or inhibitor-loaded exosomes into cardiac cells. (A) Experimental procedure for miRNA delivery using human peripheral blood-derived exosomes. Human peripheral blood-derived exosomes were loaded with miR-21 mimic or inhibitor and then used for transfection into H9C2 and HL-1 cells. Subsequent to $24 \mathrm{~h}$ treatment, the two cardiac cell lines were exposed to hypoxia for $24 \mathrm{~h}$. MiR-21 expression in (B and C) H9C2 and (F and G) HL-1 cells was measured and normalized against U6. Smad7 mRNA expression in (D) H9C2 and (H) HL-1 cells was determined using reverse transcription-quantitative polymerase chain reation. GAPDH was used as the internal control. Smad7 protein expression was detected by western blot analysis in (E) H9C2 and (I) HL-1 cells. ${ }^{*} \mathrm{P}<0.05,{ }^{* *} \mathrm{P}<0.01$ and ${ }^{* * *} \mathrm{P}<0.005$ with comparisons shown by lines. miR/miRNA, microRNA; Exo/NC, negative control miRNA-loaded exosome injection; Exo/21-M, miR-21 mimic-loaded exosome injection; Exo/21-I, miR-21 inhibitor-loaded exosome injection; Smad7, SMAD family member 7.

these results revealed that human peripheral blood-derived exosomes successfully delivered miR-21 mimics or inhibitors and modulated cardiac fibrosis in vivo. Thus, the regulation of mRNAs using miRNA-loaded exosomes derived from the human peripheral blood may represent an effective therapeutic approach against cardiac fibrosis following MI.

\section{Discussion}

MI is a public health problem and a leading cause of mortality globally (32). The development of impaired cardiac function and cardiac fibrosis due to MI serves a key function in regulating cardiac dysfunction $(6,33)$. Currently, no efficient therapies are available that may inhibit the progression of cardiac fibrosis in infarcted hearts (8). Thus, in the present study, it was examined whether miRNA delivery using human peripheral blood-derived exosomes may function as an effective therapeutic approach against MI by modulating cardiac fibrosis.

To date, a number of studies have suggested that extracellular vesicles (EVs) from in vitro cultured-cells or serum possess cardioprotective effects due to miRNAs, already present in the EVs $(34,35)$; however, these studies have focused on the function of EVs as protective vesicles as opposed to drug carriers. Notably, the present study, to the best of our knowledge, provides the first compelling evidence that human peripheral blood-derived exosomes may be successfully used as a delivery vehicle for miRNA.

miRNAs comprise a broad class of small non-coding RNAs that control the expression of complementary target mRNAs (36). Dysregulation of miRNAs by a number of mechanisms has been observed in various diseases including 

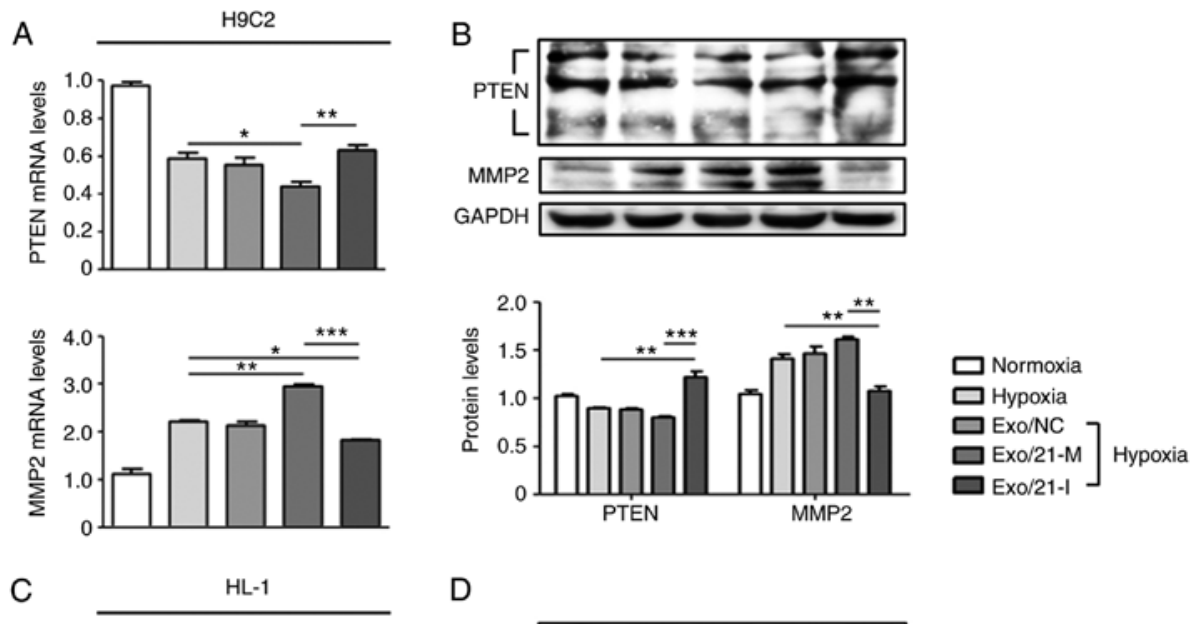

$\mathrm{D}$
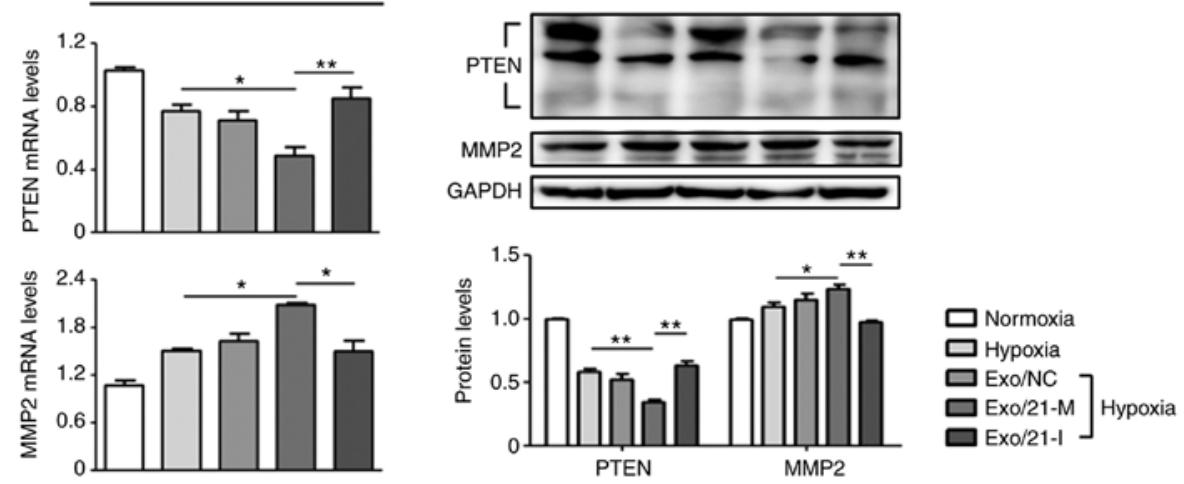

Figure 3. Effect of miR-21 mimic- or inhibitor-loaded exosomes on the modulation of target gene expression. PTEN and MMP2 expression levels following treatment with miR-21 mimic- or inhibitor-loaded exosomes were evaluated in (A and B) H9C2 and (C and D) HL-1 cells. (A and C) mRNA levels were measured using reverse transcription-quantitative polymerase chain reaction. (B and D) Protein levels were measured by western blot analysis. ${ }^{*} \mathrm{P}<0.05$, ${ }^{* *} \mathrm{P}<0.01$ and ${ }^{* * *} \mathrm{P}<0.005$ with comparisons shown by lines. miR, microRNA; PTEN, phosphatase and tensin homolog; MMP2, matrix metalloproteinase 2; Exo/NC, negative control miRNA-loaded exosome injection; Exo/21-M, miR-21 mimic-loaded exosome injection; Exo/21-I, miR-21 inhibitor-loaded exosome injection.

\section{A}

B
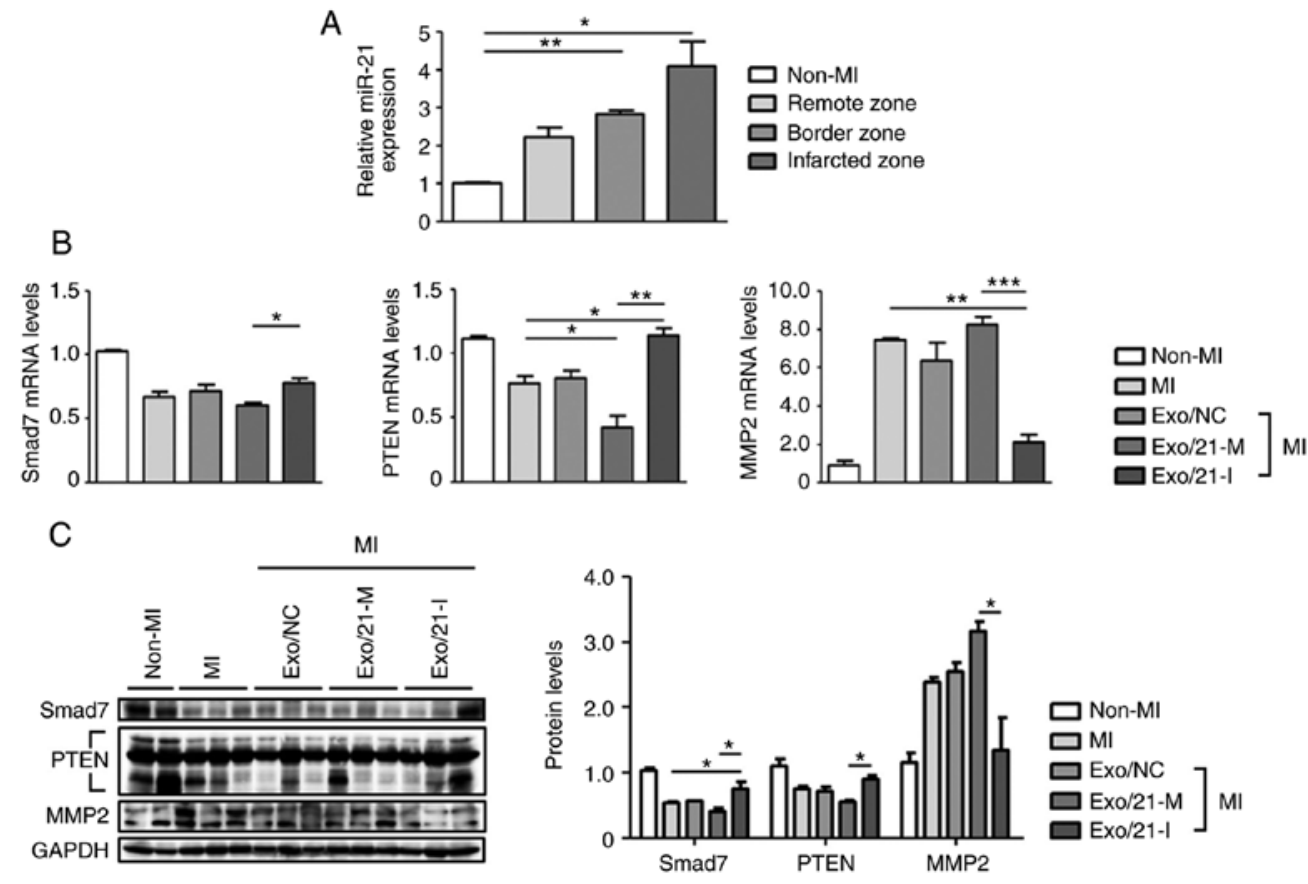

Figure 4. Delivery of miR-21 mimic- or inhibitor-loaded exosomes to MI mouse model. (A) RT-qPCR was used to determine miR-21 levels in different regions (infarcted, border zone and remote zone) of non-MI and MI mice. Relative miR-21 levels were normalized against U6. (B) Following an injection of miR-21 mimic- or inhibitor-loaded exosomes into the MI mouse model, Smad7, PTEN and MMP2 mRNA expression levels were determined usong RT-qPCR. (C) Smad7, PTEN and MMP2 protein levels were detected using western blot analysis. ${ }^{*} \mathrm{P}<0.05,{ }^{* *} \mathrm{P}<0.01$ and ${ }^{* * * *} \mathrm{P}<0.005$ with comparisons shown by lines. MI, myocardial infarction; miR, microRNA; RT-qPCR, reverse transcription-quantitative polymerase chain reaction; Smad7, SMAD family member 7; PTEN, phosphatase and tensin homolog; MMP2, matrix metalloproteinase 2; Exo/NC, negative control miRNA-loaded exosome injection; Exo/21-M, miR-21 mimic-loaded exosome injection; Exo/21-I, miR-21 inhibitor-loaded exosome injection. 

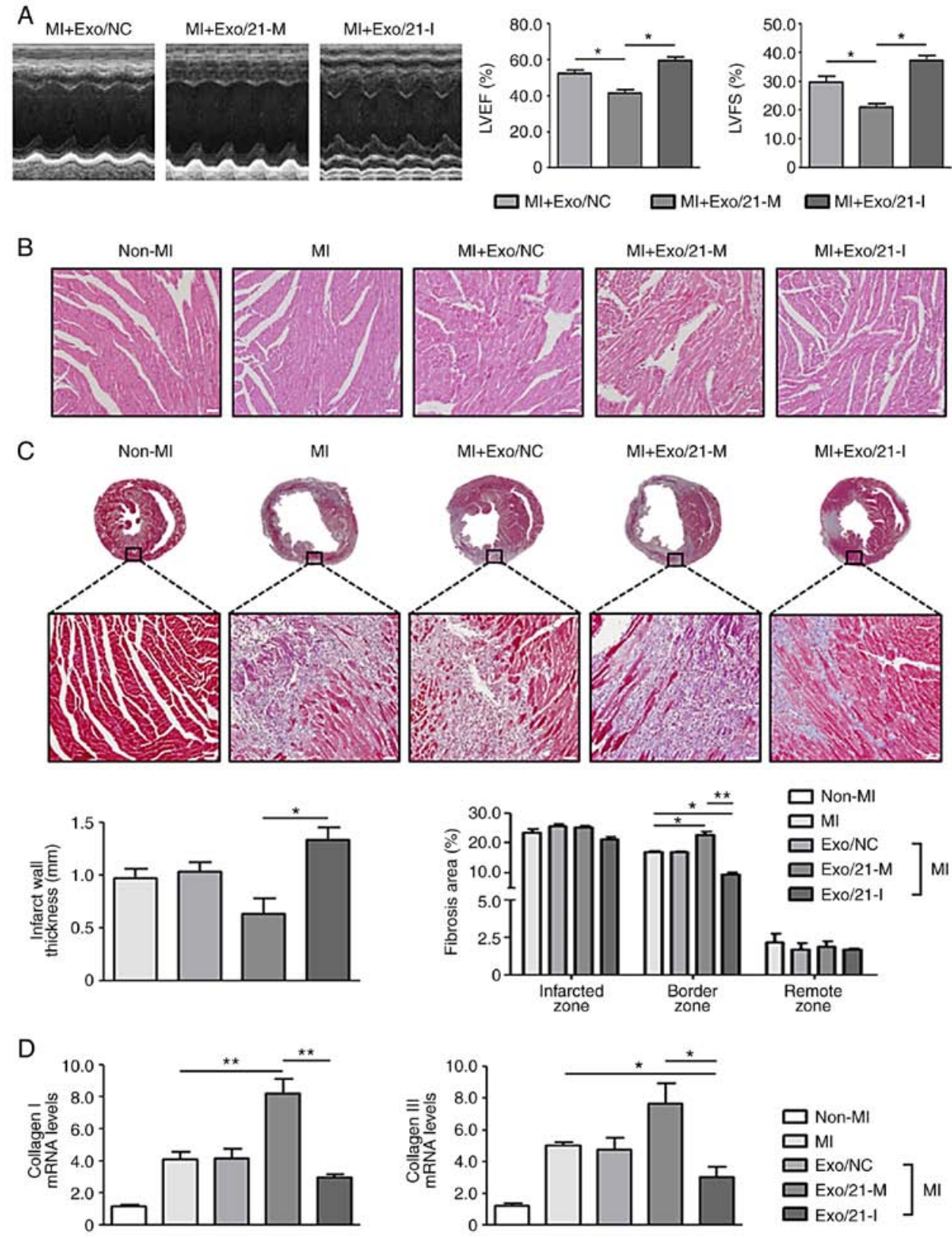

Figure 5. Effects of miR-21 mimic- or inhibitor-loaded exosomes on cardiac fibrosis in mice. (A) Representative images of M-mode echocardiography demonstrating the LV wall motion of the infarcted hearts, and the mean data of LVEF and LVFS. "P<0.05 with comparisons shown by lines. (B) Representative haemotoxylin and eosin staining in the border zone of the infarcted hearts (scale bar, $50 \mu \mathrm{m}$; magnification, x200). Pink staining indicates cardiac myocytes; blue staining indicates nuclei. (C) Representative Masson's Trichrome staining of mouse heart sections 1 week after MI (scale bar, $50 \mu \mathrm{m}$; magnification: Top row, $\mathrm{x} 2$; bottom row, $\mathrm{x} 200$ ). Blue staining indicates scar tissue; red staining indicates viable myocardium. Bar graphs exhibit the infarct wall thickness and the percentage of the fibrotic area ( $\mathrm{n}=4$ mice in each group). (D) Collagen I and III mRNA expression levels in the infarcted hearts were measured using reverse transcription-quantitative polymerase chain reaction. " $\mathrm{P}<0.05$ and ${ }^{* *} \mathrm{P}<0.01$ with comparisons shown by lines. miR, microRNA; MI, myocardial infarction; Exo/NC, negative control miRNA-loaded exosome injection; Exo/21-M, miR-21 mimic-loaded exosome injection; Exo/21-I, miR-21 inhibitor-loaded exosome injection; LVEF, left ventricular ejection fraction; LVFS, left ventricular fractional shortening.

cardiac diseases $(20,37)$. Previously, miR-21 has been revealed to serve notable functions in cardiovascular disorders (28). Furthermore, a number of potential target genes involved in miR-21-mediated cardiovascular effects have also been identified (28). For instance, miR-21 regulates the extracellular regulated kinase-mitogen activated pathway kinase signaling pathway in cardiac fibroblasts, which impacts cardiac structure and function (20). Furthermore, miR-21 serves a critical function in cardiac fibroblast activation and cardiac fibrosis following MI via the tumor growth factor- $\beta /$ Smad7 signaling pathway (19). In addition, modulation of the miR-21-regulated expression of MMP-2 via the PTEN pathway regulates cardiac fibrosis $(18,31)$. Altogether, these results confirm that miR-21 may function as a therapeutic target for MI; thus the present study examined miR-21 loaded into human peripheral blood-derived exosomes.

Exosomes are small membrane-bound vesicles (30-100 nm) secreted by most cell types. Exosomes serve a major function in cell-to-cell communication that may affect neighboring cells and distant parts of the body by transferring molecules, including proteins, mRNAs and miRNAs $(9,38,39)$. The natural biocompatibility of 
exosomes with mammalian cells suggests that they may overcome the majority of cellular barriers and drug delivery hurdles, including RNase susceptibility, endosomal accumulation, phagocytosis, multidrug resistance, cytotoxicity and immunogenicity $(40,41)$. However, exosome safety and quantity may constitute major issues in their application in clinical practice (14). As human peripheral blood has been used extensively for transfusions and is readily available, exosomes isolated from human peripheral blood may circumvent these issues. Furthermore, considering the systemic administration limitations of exosomes, including accumulation in the liver, spleen and lungs or rapid clearance by macrophages (42-44), direct injection or additional genetic modification of peripheral blood-derived exosome, as suggested by Kim et al (45), may increase the efficacy of their delivery to the heart by preventing non-specific delivery to other organs, thus increasing their therapeutic efficacy in clinical settings.

In conclusion, these results suggest that human peripheral blood-derived exosomes function as efficient vehicles for the delivery of miRNAs, which in turn may potentially be used for the treatment of cardiac diseases. This approach may be further applied to therapeutic tools for various diseases, including cancer, by delivering miRNAs other than miR-21 or diverse molecules with functionality.

\section{Acknowledgements}

Not applicable.

\section{Funding}

The present study was supported by research grants from the Basic Science Research Program through the National Research Foundation of Korea (grant no.2017R1A2B3003303), the Ministry of Education, Science and Technology (grant no. NRF-2017R1A2B3003303) and the Korean Healthcare Technology R\&D project funded by the Ministry of Health and Welfare (grant no. HI16C0058).

\section{Availability of data and materials}

The datasets used and/or analyzed during the current study are available from the corresponding author on reasonable request.

\section{Authors' contributions}

JK, HyelimP and BJ conceived and designed the experiments. JK and HyewonP performed the experiments. JK, HK and DM analyzed the data. JK, NY and BJ wrote the manuscript. All authors read and approved the final manuscript.

\section{Ethics approval and consent to participate}

The study protocol was ethically approved by the Institutional Review Board of Severance Hospital and adhered to the principles of the Declaration of Helsinki. All animal experiments were ethically approved by the Institutional Animal Care and Use Committee of Yonsei University College of Medicine (approval reference no. 2011-0136).

\section{Patient consent for publication}

Written informed consent was obtained from the subjects for participation in the present study study.

\section{Competing interests}

The authors declare that they have no competing interests.

\section{References}

1. Thygesen K, Alpert JS, Jaffe AS, Simoons ML, Chaitman BR and White HD: Third universal definition of myocardial infarction. J Am Coll Cardiol 60: 1581-1598, 2012.

2. Swynghedauw B: Molecular mechanisms of myocardial remodeling. Physiol Rev 79: 215-262, 1999.

3. Nian M, Lee P, Khaper N and Liu P: Inflammatory cytokines and postmyocardial infarction remodeling. Circ Res 94: 1543-1553, 2004.

4. Sun M, Dawood F, Wen WH, Chen M, Dixon I, Kirshenbaum LA and Liu PP: Excessive tumor necrosis factor activation after infarction contributes to susceptibility of myocardial rupture and left ventricular dysfunction. Circulation 110: 3221-3228, 2004.

5. Regula KM and Kirshenbaum LA: Apoptosis of ventricular myocytes: A means to an end. J Mol Cell Cardiol 38: 3-13, 2005.

6. van den Borne SW, Diez J, Blankesteijn WM, Verjans J, Hofstra L and Narula J: Myocardial remodeling after infarction: The role of myofibroblasts. Nat Rev Cardiol 7: 30-37, 2010.

7. Zamilpa R and Lindsey ML: Extracellular matrix turnover and signaling during cardiac remodeling following MI: Causes and consequences. J Mol Cell Cardiol 48: 558-563, 2010.

8. Fan $\mathrm{Z}$ and Guan J: Antifibrotic therapies to control cardiac fibrosis. Biomater Res 20: 13, 2016.

9. Urbanelli L, Magini A, Buratta S, Brozzi A, Sagini K, Polchi A, Tancini B and Emiliani C: Signaling pathways in exosomes biogenesis, secretion and fate. Genes (Basel) 4: 152-170, 2013.

10. Lin J, Li J, Huang B, Liu J, Chen X, Chen XM, Xu YM, Huang LF and Wang XZ: Exosomes: Novel biomarkers for clinical diagnosis. ScientificWorldJournal 2015: 657086, 2015.

11. Ha D, Yang N and Nadithe V: Exosomes as therapeutic drug carriers and delivery vehicles across biological membranes: Current perspectives and future challenges. Acta Pharm Sin B 6: 287-296, 2016.

12. Théry C, Zitvogel L and Amigorena S: Exosomes: Composition, biogenesis and function. Nat Rev Immunol 2: 569-579, 2002.

13. Batrakova EV and Kim MS: Development and regulation of exosome-based therapy products. Wiley Interdiscip Rev Nanomed Nanobiotechnol 8: 744-757, 2016.

14. Batrakova EV and Kim MS: Using exosomes, naturally-equipped nanocarriers, for drug delivery. J Control Release 219: 396-405, 2015.

15. Boon RA and Dimmeler S: MicroRNAs in myocardial infarction. Nat Rev Cardiol 12: 135-142, 2015.

16. Sun T, Dong YH, Du W, Shi CY, Wang K, Tariq MA, Wang JX and Li PF: The role of microRNAs in myocardial infarction: From molecular mechanism to clinical application. Int J Mol Sci 18, 2017.

17. Wang J, Huang W, Xu R, Nie Y, Cao X, Meng J, Xu X, Hu S and Zheng Z: MicroRNA-24 regulates cardiac fibrosis after myocardial infarction. J Cell Mol Med 16: 2150-2160, 2012.

18. Roy S, Khanna S, Hussain SR, Biswas S, Azad A, Rink C, Gnyawali S, Shilo S, Nuovo GJ and Sen CK: MicroRNA expression in response to murine myocardial infarction: miR-21 regulates fibroblast metalloprotease-2 via phosphatase and tensin homologue. Cardiovasc Res 82: 21-29, 2009.

19. Yuan J, Chen H, Ge D, Xu Y, Xu H, Yang Y, Gu M, Zhou Y, Zhu J, Ge T, et al: Mir-21 promotes cardiac fibrosis after myocardial infarction via targeting Smad7. Cell Physiol Biochem 42: 2207-2219, 2017.

20. Thum T, Gross C, Fiedler J, Fischer T, Kissler S, Bussen M, Galuppo P, Just S, Rottbauer W, Frantz S, et al: MicroRNA-21 contributes to myocardial disease by stimulating MAP kinase signalling in fibroblasts. Nature 456: 980-984, 2008.

21. Livak KJ and Schmittgen TD: Analysis of relative gene expression data using real-time quantitative PCR and the 2(-Delta Delta C(T)) method. Methods 25: 402-408, 2001. 
22. Park H, Ku SH, Park H, Hong J, Kim D, Choi BR, Pak HN, Lee MH, Mok H, Jeong JH, et al: RAGE siRNA-mediated gene silencing provides cardioprotection against ventricular arrhythmias in acute ischemia and reperfusion. J Control Release 217: 315-326, 2015.

23. Bala S, Csak T, Momen-Heravi F, Lippai D, Kodys K, Catalano D, Satishchandran A, Ambros V and Szabo G: Biodistribution and function of extracellular miRNA-155 in mice. Sci Rep 5: 10721, 2015.

24. National Research Council (US) Institute for Laboratory Animal Research: Guide for the Care and Use of Laboratory Animals. National Academies Press, Washington, DC, 1996.

25. Bartel DP: MicroRNAs: Target recognition and regulatory functions. Cell 136: 215-233, 2009.

26. Carthew RW and Sontheimer EJ: Origins and mechanisms of miRNAs and siRNAs. Cell 136: 642-655, 2009.

27. Wang B, Hao J, Jones SC, Yee MS, Roth JC and Dixon IM: Decreased Smad 7 expression contributes to cardiac fibrosis in the infarcted rat heart. Am J Physiol Heart Circ Physiol 282: H1685-H1696, 2002.

28. Cheng Y and Zhang C: MicroRNA-21 in cardiovascular disease. J Cardiovasc Transl Res 3: 251-255, 2010.

29. Oudit GY and Penninger JM: Cardiac regulation by phosphoinositide 3-kinases and PTEN. Cardiovasc Res 82: 250-260, 2009.

30. Oudit GY, Sun H, Kerfant BG, Crackower MA, Penninger JM and Backx PH: The role of phosphoinositide-3 kinase and PTEN in cardiovascular physiology and disease. J Mol Cell Cardiol 37: 449-471, 2004

31. Tao H, Zhang JG, Qin RH, Dai C, Shi P, Yang JJ, Deng ZY and Shi KH: LncRNA GAS5 controls cardiac fibroblast activation and fibrosis by targeting miR-21 via PTEN/MMP-2 signaling pathway. Toxicology 386: 11-18, 2017.

32. Chi JS and Kloner RA: Stress and myocardial infarction. Heart 89: 475-476, 2003.

33. Travers JG, Kamal FA, Robbins J, Yutzey KE and Blaxall BC: Cardiac fibrosis: The fibroblast awakens. Circ Res 118: 1021-1040, 2016.

34. Gu H, Liu Z, Li Y, Xie Y, Yao J, Zhu Y, Xu J, Dai Q, Zhong C, Zhu H, et al: Serum-derived extracellular vesicles protect against acute myocardial infarction by regulating miR-21/PDCD4 signaling pathway. Front Physiol 9: 348, 2018.

35. Khan M, Nickoloff E, Abramova T, Johnson J, Verma SK, Krishnamurthy P, Mackie AR, Vaughan E, Garikipati VN, Benedict C, et al: Embryonic stem cell-derived exosomes promote endogenous repair mechanisms and enhance cardiac function following myocardial infarction. Circ Res 117: 52-64, 2015.
36. Catalanotto $\mathrm{C}$, Cogoni $\mathrm{C}$ and Zardo G: MicroRNA in control of gene expression: An overview of nuclear functions. Int J Mol Sci 17, 2016.

37. He L, He X, Lim LP, de Stanchina E, Xuan Z, Liang Y, Xue W, Zender L, Magnus J, Ridzon D, et al: A microRNA component of the p53 tumour suppressor network. Nature 447: 1130-1134, 2007.

38. Théry C: Exosomes: Secreted vesicles and intercellular communications. F1000 Biol Rep 3: 15, 2011

39. Zhang J, Li S, Li L, Li M, Guo C, Yao J and Mi S: Exosome and exosomal microRNA: Trafficking, sorting, and function. Genomics Proteomics Bioinformatics 13: 17-24, 2015.

40. Syn NL, Wang L, Chow EK, Lim CT and Goh BC: Exosomes in cancer nanomedicine and immunotherapy: Prospects and challenges. Trends Biotechnol 35: 665-676, 2017.

41. Usman WM, Pham TC, Kwok YY, Vu LT, Ma V, Peng B, Chan YS, Wei L, Chin SM, Azad A, et al: Efficient RNA drug delivery using red blood cell extracellular vesicles. Nat Commun 9: 2359, 2018.

42. Charoenviriyakul C, Takahashi Y, Morishita M, Matsumoto A, Nishikawa $\mathbf{M}$ and Takakura Y: Cell type-specific and common characteristics of exosomes derived from mouse cell lines: Yield, physicochemical properties, and pharmacokinetics. Eur J Pharm Sci 96: 316-322, 2017.

43. Smyth T, Kullberg M, Malik N, Smith-Jones P, Graner MW and Anchordoquy TJ: Biodistribution and delivery efficiency of unmodified tumor-derived exosomes. J Control Release 199: 145-155, 2015.

44. Takahashi Y, Nishikawa M, Shinotsuka H, Matsui Y, Ohara S, Imai $\mathrm{T}$ and Takakura $\mathrm{Y}$ : Visualization and in vivo tracking of the exosomes of murine melanoma B16-BL6 cells in mice after intravenous injection. J Biotechnol 165: 77-84, 2013.

45. Kim H, Yun N, Mun D, Kang JY, Lee SH, Park H, Park H and Joung B: Cardiac-specific delivery by cardiac tissue-targeting peptide-expressing exosomes. Biochem Biophys Res Commun 499: 803-808, 2018.

This work is licensed under a Creative Commons Attribution-NonCommercial-NoDerivatives 4.0 International (CC BY-NC-ND 4.0) License. 